\section{Isolated Bloody Stools in the Moderate to Late Premature Infant. A Benign Process? Case Report and Survey}

\author{
Mariana C Baserga ${ }^{1 *}$, Joanna Johnson ${ }^{2}$, Tracy Karp² and \\ Elizabeth O'Brien ${ }^{1}$
}

'Department of Pediatrics, Division of Neonatology, University of Utah School of Medicine, Salt Lake City, Utah, USA

2Intermountain Medical Center, the LDS Hospital and Primary Children's Hospital, Intermountain Healthcare, Salt Lake City, Utah, USA

\begin{abstract}
Background: Isolated bloody stools in premature infants constitute an alarming event which could indicate necrotizing enterocolitis or other abdominal pathology. In most cases, a sepsis evaluation and abdominal X-rays (KUB) are obtained. While differences in management are anecdotally reported, they have not been studied in actual practice.

Objective: To ascertain if the clinical management of a moderate/late preterm infant (32-36 weeks gestation) presenting with isolated bloody stools differs significantly among practitioners.

Methods: Following the hospital course review of five premature infants that presented with isolated bloody stools at one institution, we assessed practice variability using an electronic survey distributed to 102 practitioners in Utah. The survey presented the clinical scenario of a healthy appearing premature infant receiving full enteral feedings with a first episode of bloody stools. Questionnaire assessed KUB frequency, use of initial laboratory tests, empiric antibiotics, Total Parenteral Nutrition (TPN) and feeding practices.

Results: 75 practitioners completed the survey: initial Complete Blood Count (CBC) and blood culture were selected by $100 \%$ and $69 \%$ of the participants, respectively. If initial KUB was normal, no further imaging was selected in $40 \%$ of practitioners while $42 \%$ performed serial KUBs. Empiric antibiotics for 48 hours were started by $48 \%$. Regarding feeding practices, $56 \%$ of practitioners stopped enteral feedings and started TPN whereas $44 \%$ continued feedings. Enteral feedings were restarted and advanced more rapidly until full feeds in $60 \%$ of responses.
\end{abstract}

*Corresponding author: Mariana Baserga, Department of Pediatrics, Division of Neonatology, University of Utah School of Medicine, Salt Lake City, PO Box 581289, Utah, USA, Tel: +1 8015877510; Fax: +1 8015857395 E-mail: mariana.baserga@hsc.utah.edu

Citation: Baserga MC, Johnson J, Karp T, O'Brien E (2015) Isolated Bloody Stools in the Moderate to Late Premature Infant. A Benign Process? Case Report and Survey. J Neonatol Clin Pediatr 2: 012.

Received: March 26, 2015; Accepted: May 19, 2015; Published: June 02, 2015
Conclusion: Most surveyed practitioners evaluated a premature infant presenting with a first episode of bloody stools with an initial $\mathrm{CBC}$ and KUB. However, only half of the practitioners would stop enteral feedings and only a third would start TPN. Moreover, empiric antibiotics were ordered only by half of the participants. Return to full enteral feedings was achieved more rapidly. We speculate that there is a trend among practitioners to treat isolated bloody stools in this population as a benign condition.

Keywords: Isolated bloody stools; Moderate or Late preterm infant

\section{Introduction}

Isolated bloody stools in an otherwise healthy looking late (34-36 weeks Gestational Age (GA)) or moderate (32-33 weeks GA) premature infant constitutes an alarming event which could indicate intraabdominal pathology or, in the worst case scenario, Necrotizing Enterocolitis (NEC). In such cases, most practitioners perform a routine screen or evaluation for infection/sepsis, including white blood cell count and blood culture, and obtain one or serial abdominal radiographs. Very often, bacterial culture and viral studies in stool are also included as part of the diagnostic work up. In terms of treatment approach, management differs significantly from center to center and among practitioners. Usually feedings are discontinued (NPO status), gastric decompression initiated, and empiric antibiotic treatment started.

In spite of the fact that there seems to be a perceived increase in the incidence of isolated bloody stools $(3.5-8.8 \%)$, there is a paucity of literature addressing management of these patients $[1,2]$. The objective of the present report is to describe the hospital course of five moderately premature infants (32-33 5/7 weeks GA at birth) that presented with isolated bloody stools at our institution. Based on our own observation of the variability in the management of these infants, we conducted a survey among neonatal care practitioners in healthcare centers in the State of Utah to assess the present approach to this condition. The objective of the survey was to ascertain if the clinical management of moderate to late premature infants that present with an isolated bloody stool but are otherwise asymptomatic differs significantly among practitioners.

\section{Methods}

\section{Case reports}

Medical charts of five premature infants (32-33 5/7 weeks GA at birth) born at Intermountain Medical Center (Murray, UT) and that presented with isolated bloody stools in the Neonatal Intensive Care Unit (NICU) in that institution during the year 2010 were reviewed. Institutional board review was consulted and consent waiver was obtained.

\section{Survey}

An electronic 10 question survey was conducted via e-mail to include 102 practitioners in Utah. Neonatal nurse practitioners, general pediatricians and neonatologists (training fellows and board certified neonatologists) working in a range of practices (private, academic, level I-III neonatal intensive care units) were invited to participate. 
Citation: Baserga MC, Johnson J, Karp T, O'Brien E (2015) Isolated Bloody Stools in the Moderate to Late Premature Infant. A Benign Process? Case Report and Survey. J Neonatol Clin Pediatr 2: 012.

\begin{tabular}{|c|c|c|c|c|c|}
\hline & Case 1 & Case 2 & Case 3 & Case 4 & Case 5 \\
\hline Gestational age (weeks) & 32 & 33 & $324 / 7$ & $335 / 7$ & $332 / 7$ \\
\hline Birth weight (g) & 1560 & 1920 & 1750 & 1320 & 1760 \\
\hline Age at event (days) & 10 & 23 & 12 & 17 & 17 \\
\hline Feedings at time of event & $22 \mathrm{kcal} / \mathrm{oz}$ of Similac Special Care & $24 \mathrm{kcal} / \mathrm{oz}$ of breast milk & $22 \mathrm{kcal} / \mathrm{oz}$ of breast milk & $24 \mathrm{kcal} / \mathrm{oz}$ of breast milk & $24 \mathrm{kcal} / \mathrm{oz}$ of breast milk \\
\hline \multicolumn{6}{|l|}{ Laboratory } \\
\hline $\begin{array}{l}\text { WBC count }(1000 / \mu \mathrm{l}) / \\
\text { differential }\end{array}$ & $\begin{array}{l}6800 / 35 \% \text { neutrophils, } 14 \% \\
\text { bands, } 1 \% \text { eosinophils }\end{array}$ & $\begin{array}{l}15600 / 41 \% \text { neutro- } \\
\text { phils, } 3 \% \text { eosinophils }\end{array}$ & $\begin{array}{l}9300 / 36 \% \text { neutrophils, } \\
2 \% \text { eosinophils }\end{array}$ & $\begin{array}{l}8200 / 2 \% \text { bands, } 23 \% \\
\text { neutrophils, } 1 \% \text { eosin- } \\
\text { ophils }\end{array}$ & $\begin{array}{l}15800 / 39 \% \text { neutrophils, } \\
4 \% \text { eosinophils }\end{array}$ \\
\hline Platelet count $(1000 / \mu \mathrm{l})$ & 211,000 & 460,000 & 304,000 & 333,000 & 353,000 \\
\hline $\begin{array}{l}\text { Duration of bloody } \\
\text { stools (days) }\end{array}$ & 1 & 1 & 1 & 1 & 1 \\
\hline Time without feeds(days) & 5 & 4 & 5 & 7 & 2 \\
\hline $\begin{array}{l}\text { Length of antibiotic } \\
\text { treatment (days) }\end{array}$ & 3 & 7 & 2 & 7 & 2 \\
\hline $\begin{array}{l}\text { Change in type of } \\
\text { feeding }\end{array}$ & no & no & no & no & no \\
\hline $\begin{array}{l}\text { Recurrent episode } \\
\text { before discharge }\end{array}$ & $\begin{array}{l}\text { Yes (blood present in } 1 \text { stool at } 28 \\
\text { days of life) }\end{array}$ & no & no & no & no \\
\hline
\end{tabular}

Table 1: Clinical characteristics of 5 premature infants with isolated blood in stools.

The survey included the following general background questions: training specialty (neonatology, pediatrics, neonatology nurse practitioner, neonatology fellow); years of practice in the NICU (less than 5,5 to 10 , or more than 10 years), type of hospital that best described responder's practice (university hospital, private practice, community hospital), NICU level (I, II or III).

The case scenario presented in the survey was of an otherwise healthy looking moderate to late premature infant receiving enteral (oral/gavage) feeds with breast milk, fortified breast milk, and/or premature formula, and that presented with a first episode of frank bloody stools. Respondents chose one or more of several preferred actions for each management question.

Options for initial laboratory assessment included Complete Blood Cell count (CBC), blood culture, stool culture for bacteria, Rotavirus, Enterovirus, Adenovirus, Clostridium difficile. Regarding imaging, optional answers included: 1) initial abdominal X-ray, and if normal no further imaging; 2) initial abdominal X-ray, and even if normal, serial studies until no further bloody stools are present; 3) serial abdominal X-ray studies for 24 hours; 4) serial abdominal X-ray studies for 48 hours; or 5) no imaging studies obtained.

In terms of feeding practices, possible answers were as follows: 1) Infant is made NPO until there are no more bloody stools; 2) Infant is made NPO for at least 2-3 days; 3 ) Infant is made NPO for at least 3-5 days; 4) Infant remains NPO for at least 5-7 days; 5) Infant remains NPO for $>7$ days; or 6) PO feedings are continued. If making the patient NPO for any period of time, we inquired if the practitioner orders Total Parenteral Nutrition (TPN).

Option for modalities to restart enteral feedings included: 1) $\mathrm{PO} /$ gavage feedings are restarted at small volumes and advance slowly (20 mL/kg/day) until reaching full feedings; 2) Feedings are restarted and advanced more rapidly $(40-60 \mathrm{~mL} / \mathrm{Kg} /$ day $)$ until back to full feeds; 3) Feedings are restarted at full target volume; 4) The same type of feedings the baby was on are resumed (breast milk, fortified breast milk or premature formula); 5) A different formula is started; and/or 6) An elemental-type formula is preferred.

Regarding empiric antibiotic use, optional answers included: 1) Empiric antibiotics are prescribed; 2) Empiric antibiotics are continued for a 48-72 hour rule out sepsis. If blood cultures are negative, antibiotics are discontinued; 3 ) Antibiotics are continued for 5-7 days; 4) Antibiotics are continued for $>7$ days; or 5) Empiric antibiotics are not started.

\section{Results}

\section{Case reviews}

The characteristics of the five patients are described in table 1 . In summary, the birth GA of the patients ranged between $320 / 7$ and $335 / 7$ weeks, birth weight mean was $1662 \pm 229$ grams. There were four males and one female. The age at bloody stool event ranged from 10 to 23 days of life. All five babies were on full enteral feedings, 4/5 infants were receiving fortified breast milk, the remaining infant was receiving Similac Special Care ${ }^{\bullet} 20$ calorie/oz (SSC) or SSC ${ }^{\oplus}$ (22 calories/oz). Breast milk fortification was achieved using Similac Human Milk Fortifier (powder). Following the first bloody stool episode, all five infants had at least an initial abdominal X-ray. Four of the initial abdominal X-rays were read as normal without signs of pneumatosis intestinalis. In one infant the initial abdominal X-ray had a suspicious area of possible pneumatosis intestinalis that resolved in a follow up abdominal X-ray that same day. Serial abdominal X-rays were performed every six hours in all infants for the first 24 hours, then every 12 hours for 1-2 days, then discontinued. 
Initial CBC was within normal limits in four babies. In Case 1 there were $14 \%$ bands. No significant eosinophilia was observed in any of the patients. Platelet count was within normal limits in all babies. C-Reactive Protein (CRP) was $<0.5$ in all five infants. CBC and CRP were repeated in all infants the following day and then discontinued.

Antibiotic treatment was started in all cases (Vancomycin and Gentamicin in four infants, Ampicillin and Gentamicin in one infant) and continued for a median of three days (2-7 days). Initial blood cultures were negative in $4 / 5$ patients but was positive in one infant (Staphylococcus capitis). Stool viral cultures for Rotavirus, Adenovirus and Enterovirus were negative in all 5 infants. Gastrointestinal decompression with suction was not required in these infants since they did not exhibit abdominal distention or signs of ileus. Duration of bloody stools was one day ( 1 or several stools contained blood during that first day) and time without feeds was a median of five days (2-7 days) during which all infants received Total parenteral nutrition. When enteral feedings were restarted, breast milk (four babies) or SSC 20 calories/oz (one baby) were used initially at $20 \mathrm{~mL} / \mathrm{kg} / \mathrm{day}$, and volume feedings advanced rapidly back to full intake of $150 \mathrm{~mL} / \mathrm{kg} /$ day within $2-4$ days. Fortification to 22 or 24

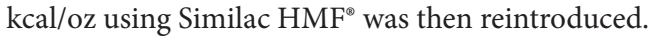

One infant had a recurrent bloody stool episode on day of life 28. CBC, CRP and abdominal X-ray evaluations were normal, a blood culture was therefore not sent nor were antibiotics started. The infant remained NPO for one day and then feeds were resumed at $60 \mathrm{~mL} / \mathrm{kg} /$ day and advanced to ad lib demand without difficulty. The infant was discharged home on Neosure $22 \mathrm{kcal} / \mathrm{oz}$ formula.

\section{Survey}

Demographics: We obtained responses from 75 surveyed practitioners (73\% response rate). There were 35 Neonatal Nurse Practitioners, 29 neonatologists, eight general pediatricians, and three neonatology fellows. Forty six percent (46\%) of practitioners had more than 10 years of practice, $28 \%$ had between $5-10$ years; and $26 \%$ had less than five years.

Forty three percent (43\%) reported practicing exclusively in an academic setting, $25 \%$ in a community type hospital, and $31 \%$ described a combination of academic and community hospital setting. Ninety percent (90\%) of participants reported working in a Level 3-4 NICU, $27 \%$ in a Level 2 NICU, and $10 \%$ also provided services in a well baby nursery setting.

Clinical scenario management responses: Table 2, displays responses for the presented clinical scenario of an otherwise healthy appearing premature infant that was receiving oral/gavage feeds and that presented with a first episode of frank bloody stools.

In the laboratory tests category, more than one response could be selected: CBC and blood culture were selected by $100 \%$ and $69 \%$ of the participants, respectively. There was a wide variation in practice regarding stool microbiologic studies (viral/bacterial).

The option of ordering an initial abdominal X-ray and no further imaging if the initial study was deemed normal was selected in $40 \%$ of cases. The options of ordering serial abdominal X-rays until no further bloody stools are present or for at least 24 hours following initial imaging study were chosen by $32 \%$ and $25 \%$ of practitioners respectively. Of importance, all practitioners did chose to obtain at least one abdominal X-ray.

\begin{tabular}{|c|c|}
\hline \multicolumn{2}{|l|}{ Practitioners $(\mathrm{N}=75) \mathrm{n}(\%)$} \\
\hline Initial Laboratory Tests (selected all that apply) & No response: $3(4)$ \\
\hline $\mathrm{CBC}$ & $72(100)$ \\
\hline Blood Culture & $50(69)$ \\
\hline Stool Culture & $13(18)$ \\
\hline Rotavirus (culture or rapid type of test) & $25(34)$ \\
\hline Enterovirus (culture or rapid type of test) & $19(26)$ \\
\hline Adenovirus (culture or rapid type of test) & $19(26)$ \\
\hline Clostridium Difficile toxine (s) & $4(6)$ \\
\hline Other & $18(25)$ \\
\hline Abdominal Imaging Studies & No response: $2(2)$ \\
\hline Initial abdominal X-Ray, if normal no further imaging & $29(40)$ \\
\hline $\begin{array}{l}\text { Initial abdominal X-Ray, serial studies until no further bloody } \\
\text { stools }\end{array}$ & $23(32)$ \\
\hline Serial abdominal X-Ray studies for 24 hours & $19(25)$ \\
\hline Serial abdominal X-Ray studies for 48 hours & $2(3)$ \\
\hline I do not obtain imaging studies & $0(0)$ \\
\hline Management of Feedings right after bloody stool episode & No response: 1 (1) \\
\hline Infant is made NPO until there are no more bloody stools & $23(31)$ \\
\hline Infant remains NPO for at least 2-3 days & $22(29)$ \\
\hline Infant remains NPO for at least 3-5 days & $2(2)$ \\
\hline Infant remains NPO for at least 5-7 days & $2(2)$ \\
\hline Infant remains NPO for $>7$ days & $3(4)$ \\
\hline PO feedings are continued & $22(29)$ \\
\hline Total Parenteral Nutrition & No response: $1(1)$ \\
\hline Never & $30(40)$ \\
\hline
\end{tabular}

Table 2: Responses to management of case scenario by surveyed practitioners.

Regarding feeding practices, $68 \%$ of practitioners would stop oral/gavage feedings following a bloody stool episode. One third of the surveyed practitioners would restart feeds when no blood was noted in stools, or after 48-72 hours of NPO status. Interestingly, another third of the practitioners would chose to continue oral/gavage feedings when presented with this particular scenario. In case of making patient NPO, only a third of the responders would routinely start total parenteral nutrition.

In the survey, enteral feedings would be restarted and advanced more rapidly ( $40 \mathrm{~mL} / \mathrm{kg} /$ day) until full feeds in $56 \%$ of cases, $32 \%$ chose to use the same type of feedings the patient was on before the bloody stool episode, while $9 \%$ would chose an elemental type formula. Empiric antibiotic therapy for 48 hours would be started by $53 \%$ of practitioners. Only 2\% would continue antibiotics for 5-7 days. Of interest, $44 \%$ of participants would not choose to start empiric antibiotics.

\section{Discussion}

In this report we described the hospital course of five premature infants (32 0/7 and $335 / 7$ weeks GA) that presented with isolated bloody stools at our institution. These five case reports depict a very common occurrence in many level 2-3 NICUs and much discussion typically surrounds decision making [2,3]. Questions such as laboratory and imaging needs, indications to withhold enteral feedings and for how long, the need to start antibiotics or not and for how long to continue them are among the most common. This lead us to inquire what other practitioners would do when faced with this common scenario. As we had initially speculated, there seems to be 
significant variability in the management approach of the moderate/late premature infant that presents with isolated bloody stools.

In spite of the perceived increased incidence of isolated bloody stools in premature babies, few clinical reports are available $[1,3]$. One of the most detailed reports is by Maayan-Metzger et al., who described the characteristics and medical management of 103 preterm infants with isolated bloody stools and normal abdominal $\mathrm{X}$-rays during a 12 year retrospective study in the same institution in Israel [4]. The authors analyzed 2 time periods before and after the introduction of a more liberal approach (shortened NPO and antibiotic use) in the treatment of isolated bloody stools. They found that the rate of isolated bloody stools, the use of antibiotics and feeding cessation decreased in the second period in premature infants. Importantly, the authors attributed the decreased rate of bloody stools to the significant increase in breast milk feedings in the second period. With the more liberal approach, there was no increase in complications following the episode of bloody stools.

The most common differential diagnoses once NEC is ruled out (negative KUB for pneumatosis intestinalis, no evidence of ileus) includes the presence of anal and rectal fissures, ecchymotic colitis, viral Gastroenteritis (GE) and/or milk feeding (protein) intolerance [5-8]. A thorough exam is warranted in all patients presenting with blood in the stools and if a diagnosis of anal fissure is made and abdominal films are normal, then a conservative approach with symptomatic treatment is recommended. Symptoms are typically self-resolving but may reoccur. It is important to point out that deeper rectal fissures can be easily missed and therefore the real incidence of GI fissures remains uncertain [6]. The opposite scenario (anal fissures "masking" NEC) should also be considered [9].

Viral GE secondary to organisms such as Rotavirus, Adenovirus and Enterovirus can in some instances present with isolated bloody stools, relatively normal KUBs and benign laboratory and physical findings $[7,8,10]$.

Milk protein intolerance is a more complicated diagnosis to make, especially in premature infants. The incidence of protein allergy early in life is low, $2-3 \%$ in developed countries, and has also been reported in premature infants but is rare [11,12]. Allergic enterocolitis can present as recurrent NEC [13]. Family history of milk protein allergy can be helpful in establishing a diagnosis. Patients may present with isolated bloody stools but no other symptoms of intolerance such as abdominal distention, vomiting and/or diarrhea. Furthermore, both premature and term infants do not typically present with cutaneous or respiratory symptoms observed in older infants. Blood laboratory findings associated with this disorder may include elevated IgE, microcytic anemia and the presence of eosinophilia. However these findings are very nonspecific and can be absent $[14,15]$. In the future, advanced diagnostic testing using microarray technology and epitope analysis may improve the diagnostic accuracy of milk protein allergy by determination of specific IgE against specific allergen components of cow's milk protein [15]. The basic treatment (and most times the best diagnostic tool) of milk protein intolerance is avoidance of milk protein containing products. In neonates a milk substitute such as hydrolyzed formulas are recommended. In case of intolerance to extensively hydrolyzed formulas, a formula based on amino acids can be suggested.

As in many case scenarios in babies with isolated bloody stools, none of these differential diagnoses applied to the 5 cases presented from our institution. In the 5 patients, duration of blood in the stool was $<24$ hours, time of NPO ranged from 2 to up to 7 days, all viral studies were negative, abdominal $\mathrm{x}$-rays were non-specific and only one of the infant's had recurrence of bloody stool at 28 days. In all cases, an attempt was made to reinitiate feedings using breast milk or formula. All patients tolerated this approach and therefore milk protein allergy was excluded by clinical response. In the absence of a strong family history, it would seem safe and fair to challenge these infants without using dietary restrictions; however should symptoms reoccur substitution with a hydrolyzed formula should be considered. A small randomized study by Arvola et al., found that in patients with isolated bloody stools elimination of cow's milk protein in 19 of 39 infants did not reduce the length of bloody stools [16].

A possible, but less understood, entity termed "ecchymotic colitis" can present in the same manner. These neonates typically are asymptomatic except for the presence of blood in the stool. The etiology remains unknown but endoscopic and Histopathological studies showed patches of hemorrhagic and inflammatory lesions in the rectum, sigmoid and/or colon amid normal mucosa [5,16-18]. In a report by Cordero et al., 19/46 (41\%) of the patients with isolated blood in the stool were deemed to have isolated rectal bleeding with ecchymotic colitis [19]. Unfortunately, performing an invasive procedure such as endoscopy with biopsy to establish this diagnosis is not an option since this is a self-limited condition with a very benign course. Therefore, hemorrhagic colitis also remains a diagnosis of suspicion in our reported cases. Lastly, despite universal use of vitamin $\mathrm{K}$ for prophylaxis of vitamin $\mathrm{K}$ deficiency bleeding (formerly known as "hemorrhagic disease of the newborn") in both term and preterm infants, coagulation disturbances should be part of the differential diagnosis in patients that present with isolated bloody stools, in particular if there is history of no vitamin K prophylaxis [20].

Survey findings included a wide variability in treatment of the well-appearing, moderate premature infant on full enteral feedings who present with a first episode of bloody stools. The majority of respondents would obtain a $\mathrm{CBC}$ and a KUB, half of the respondents would make the baby NPO for any duration of time and start empiric antibiotics, and only a third would start TPN. Return to full enteral feedings would be achieved quite rapidly and, in most cases, using the original type of nutrition. The majority of practitioners would order empiric antibiotics, for an average of 48-72 hours of treatment as long as cultures remained negative. Interestingly, a significant proportion of surveyed practitioners would not start antibiotics based on diagnostic criteria as presented in the survey. Follow up to this survey could include the type of antibiotics used to manage presumed sepsis/NEC, but would likely reveal the same variability in treatment.

A limitation of this review includes the lack of long-term follow up after the infants' discharge from the hospital. It is unknown at this time if these patients had further feeding problems or recurrent blood in the stool. Further evaluation of the type of human milk fortifier would also be beneficial to delineate if powder vs liquid fortifiers and the type of protein would be better tolerated at increasing nutrient and caloric densities for this population. During the current case evaluations a powder fortifier was used. Liquid fortifier has since been introduced without an observed increase in feeding intolerance or bloody stools to date.

In conclusion, most surveyed practitioners in Utah evaluate a healthy, moderate to late well appearing premature infant receiving full enteral feedings who presents with a first episode of bloody stools 
with limited tests. Only half of the surveyed practitioners would make the baby NPO and only a third would start TPN. Moreover, empiric antibiotics are ordered only by half of the participants. Return to full enteral feedings is achieved more rapidly and in most instances using the original type of nutrition. We speculate that there is a trend among practitioners to treat isolated bloody stools in this population as a benign condition, with cautious 48-72 hour evaluation period and a rapid return to baseline enteral feedings. Based on the practice variability encountered in this report, this work could become the basis of a quality improvement project with a goal to standardize the approach and management of isolated bloody stools in the moderate/late premature infant.

\section{References}

1. Gordon PV, Swanson JR, Attridge JT, Clark R (2007) Emerging trends in acquired neonatal intestinal disease: is it time to abandon Bell's criteria? J Perinatol 27: 661-671.

2. Maayan-Metzger A, Ghanem N, Mazkereth R, Kuint J (2004) Characteristics of neonates with isolated rectal bleeding. Arch Dis Child Fetal Neonatal Ed 89: 68-70.

3. Giacoia GP, Williams GP (1995) Rectal bleeding due to nonspecific colitis in premature infants. South Med J 88: 789-791.

4. Maayan-Metzger A, Schushan-Eisen I, Kuint J (2010) Management of isolated rectal bleeding in newborn infants: comparison of two time periods. Acta Paediatr 99: 215-218.

5. Canioni D, Pauliat S, Gaillard JL, Mougenot JF, Bompard Y, et al. (1997) Histopathology and microbiology of isolated rectal bleeding in neonates: the so-called 'ecchymotic colitis'. Histopathology 30: 472-477.

6. Thompson EC, Brown MF, Bowen EC, Smith LM, vander Griten D (1996) Causes of gastrointestinal hemorrhage in neonates and children. South Med J 89: 370-374.

7. Sharma R, Hudak ML, Premachandra BR, Stevens G, Monteiro CB, et al. (2002) Clinical manifestations of rotavirus infection in the neonatal intensive care unit. Pediatr Infect Dis J 21: 1099-1105
8. Bagci S, Eis-Hübinger AM, Yassin AF, Simon A, Bartmann P, et al. (2010) Clinical characteristics of viral intestinal infection in preterm and term neonates. Eur J Clin Microbiol Infect Dis 29: 1079-1084.

9. Bowen A 3rd (1980) Mild form of neonatal necrotizing enterocolitis masked by anal fissures. Radiology 137: 657-659.

10. Ronchi A, Doern C, Brock E, Pugni L, Sánchez PJ (2014) Neonatal adenoviral infection: a seventeen year experience and review of the literature. J Pediatr 164: 529-535.

11. Church JA, Wang DW, Swanson V, Thomas D, Sinatra F (1978) Cow's milk allergy in a premature infant with hypereosinophilia and hyperimmunoglobulinemia E. Ann Allergy 41: 307-310.

12. Bahna SL (2002) Cow's milk allergy versus cow milk intolerance. Ann Allergy Asthma Immunol 89: 56-60.

13. Srinivasan P, Brandler M, D'Souza A, Millman P, Moreau H (2010) Allergic enterocolitis presenting as recurrent necrotizing enterocolitis in preterm neonates. J Perinatol 30: 431-433.

14. Machida HM, Catto Smith AG, Gall DG, Trevenen C, Scott RB (1994) Allergic colitis in infancy: clinical and pathologic aspects. J Pediatr Gastroenterol Nutr 19: $22-26$.

15. Host A, Halken S (2014) Cow's milk allergy: where have we come from and where are we going? Endocr Metab Immune Disord Drug Targets 14: 2-8.

16. Arvola T, Ruuska T, Keränen J, Hyöty H, Salminen S, et al. (2006) Rectal bleeding in infancy: clinical, allergological, and microbiological examination. Pediatrics 117: 760-768.

17. Dupont C, Badoual J, Le Luyer B, Le Bourgeois C, Barbet JP, et al. (1987) Rectosigmoidoscopic findings during isolated rectal bleeding in the neonate. J Pediatr Gastroenterol Nutr 6: 257-264.

18. Anveden-Hertzberg L, Finkel Y, Sandstedt B, Karpe B (1996) Proctocolitis in exclusively breast-fed infants. Eur J Pediatr 155: 464-467.

19. Cordero L, Giannone PJ, Valentine CJ, Coley BD, Nankervis CA (2012) Pneumatosis coli: A benign form of necrotizing enterocolitis. JNPM 3: 221229.

20. Shearer MJ (2009) Vitamin K Deficiency Bleeding (VKDB) in early infancy. Blood Rev 23: 49-59. 